\title{
PLANET-DISK SYMBIOSIS
}

\author{
Re'em Sari and Peter Goldreich \\ California Institute of Technology, MS 130-33, Pasadena, CA 91125 \\ Received 2003 July 5; accepted 2004 March 18; published 2004 April 5
}

\begin{abstract}
Planets form in disks around young stars. Interactions with these disks cause them to migrate and thus affect their final orbital periods. We suggest that the connection between planets and disks may be deeper and involve a symbiotic evolution. By contributing to the outward transport of angular momentum, planets promote disk accretion. Here we demonstrate that planets sufficiently massive to open gaps could be the primary agents driving disk accretion. Those having masses below the gap opening threshold drift inward more rapidly than the disk material and can only play a minor role in its accretion. An even more intimate symbiosis involving gap opening planets may result if they acquire most of their mass prior to gap formation. Given a small initial eccentricity, just a fraction of a percent, the orbital eccentricity of a massive planet may grow rapidly once a mass in excess of the planet's mass has been repelled to form a gap around the planet's orbit. Then, as the planet's radial excursions approach the gap's width, subsequent eccentricity growth slows so that the planet's orbit continues to be confined within the gap.
\end{abstract}

Subject headings: planetary systems: formation — planetary systems: protoplanetary disks

\section{INTRODUCTION}

One of the most important scientific developments of the nineties was the discovery of extrasolar planets (Mayor \& Queloz 1995; Marcy \& Butler 1998), planets that orbit other stars. Currently, more than 100 are known, along with about a dozen systems containing more than one planet. The basic data, i.e., masses and orbits, have revealed two major surprises: (1) Jovian mass planets with short period orbits and (2) isolated planets with large orbital eccentricities.

Current planet formation theories fail to account for the formation of giant planets on short period orbits. Instead, there is a general consensus that these planets migrated inward from where they were born. Theoretical work done in the eighties and nineties established that angular momentum and energy exchanged between planet and disk at discrete Lindblad resonances would result in the rapid migration of the planet (Goldreich \& Tremaine 1980), and that almost invariably the migration would be inward (Ward 1986; Artymowicz 1993a, 1993b). In turn, for sufficiently massive planets, this exchange would modify the disk's density profile. A familiar aspect of the latter phenomenon is the opening of a gap around the orbit of a massive planet. This locks the orbital evolution of the planet to that of the disk, a behavior referred to as type II migration (Ward 1997).

\section{ACCRETION}

Disk accretion is driven by the outward transport of angular momentum. The mechanism by which this is accomplished in protostellar disks is uncertain. Molecular viscosity is far too small to be effective. Magnetorotational instability is a more plausible candidate, but the disk's electrical conductivity may be inadequate to sustain it.

Here we examine the possibility that torques from embedded planets drive disk accretion. This idea is not new. Stimulated by Larson's (Larson 1989) suggestion that spiral waves might drive disk accretion, Goodman \& Rafikov (2001) proposed that these waves could be excited by planets with masses too small to open gaps. However, they ignored the migration of the planets. A general argument given below establishes that subcritical planets would disappear before significant disk accretion could take place.

The critical mass required for gap formation in a disk without any intrinsic viscosity is less than $1 M_{\oplus}$ (Hourigan \& Ward 1984; Ward \& Hourigan 1989; Rafikov 2002). Thus, subcritical planets are composed of elements heavier than helium that comprise a fraction $f$, on the order of a percent, of the disk's mass. Through the torques they exert at Lindblad resonances, these planets transfer angular momentum outward from material interior to their orbits to that external to their orbits. The dominant resonances are located about a distance $h$, the scale height of the disk, inward and outward from the planet's orbit. Therefore, these two rates are almost equal. Only the small fractional difference, on the order of $h / r$, causes the planet to drift inward. Thus, each planet transfers angular momentum across its orbit at a rate that is larger by a factor $r / h$ than that at which its angular momentum decays.

Although the major planet-disk interactions occur at distance $h$ from the planet's orbit, density waves carry the angular momentum farther away and deposit it at distance $\lambda$, where typically $h<\lambda<r$. So the contribution from each planet to the local luminosity of angular momentum through the disk is a factor $\lambda / h$ greater than the rate at which it loses angular momentum. If subcritical planets were the sole source of the disk's angular momentum luminosity, the timescale for planet accretion would be related to that for disk accretion by a factor $\approx(\lambda / h) f$. Goodman \& Rafikov (2001) estimate $\lambda / h \sim 2$ for Earth-sized planets. Since the total mass of subcritical planets is at most a percent of the disk mass $(f<0.01)$, they could not be responsible for the accretion of more than $2 \%$ of the disk mass.

Although subcritical planets are unable to drive disk accretion, massive planets might. They reside in gaps and accrete at the same rate as the surrounding disk material. If they were the sole source of disk accretion, their number density and masses would affect the accretion rate but be irrelevant to the final outcome. Suppose there were one giant planet per logarithmic radius interval. Then gap widths would be on the order of the radius, and one-sided torques from individual planets would have approximate magnitudes given by 
$\left(M_{p} / M_{*}\right)^{2} \Sigma r^{2}(\Omega r)^{2}$, where $M_{p}$ and $M_{*}$ are the masses of the planet and the star, $\Sigma$ is the disk's surface density, $\Omega$ is the orbital angular velocity, and $r$ is the orbital radius. Under these conditions, the angular momentum luminosity in the disk would be on the order of the one-sided torque. This would yield an accretion timescale on the order of $\left(M_{*} / M_{p}\right)^{2} \Omega^{-1}$ provided $M_{p} \leq \Sigma r^{2}$. Note that this timescale is independent of the disk mass and demonstrates that Jupiter-sized planets could cause a disk to accrete in about $10^{6} \mathrm{yr}$. Higher rates would occur if the planets were more massive or numerous, subject to the constraint that the mass in planets does not exceed the disk mass.

\section{ECCENTRICITIES AND GAPS}

We adopt standard parameters for use in numerical evaluations. They are $M_{d} / M_{*}=10^{-2}$ for disk-to-star mass ratio, where $M_{d}$ is the disk mass within the planet's orbit, $\mu=$ $M_{p} / M_{*} \approx 10^{-3}$ for the planet-to-star mass ratio, and $h / r \approx$ 0.04 for the disk aspect ratio. Also, in this section, we treat the viscosity with the customary approximation and choose $\alpha=10^{-4}$, which, combined with our disk mass and scale height, sets a mass accretion rate $\dot{M} \approx 10^{-8} M_{\odot} \mathrm{yr}^{-1}$. This choice is consistent with observational determinations of accretion rates onto $1 \mathrm{Myr}$ old $\mathrm{T}$ Tauri stars (Hartmann et al. 1998). Higher accretion rates are typical at earlier stages, but any giant planets that might have formed then would have been consumed by their parent stars. As we are concerned with planets that survived, it is the later accretion stages that are relevant to our investigation. If, as we speculate in the previous section, there is no intrinsic viscosity and accretion is entirely driven by planet torques, then the effective $\alpha$ may be even lower than the value adopted above.

Goldreich \& Sari (2003, hereafter GS03) suggest that planetdisk interactions might have given rise to the large eccentricities of extrasolar planets. The important interactions are those at Lindblad and corotation resonances. For sufficiently small eccentricity, the interactions are linear. In this limit, those at ordinary Lindblad resonances, which excite eccentricity, are slightly less effective than those at corotation resonances, which damp it. However, nonlinear saturation of the corotation resonances occurs at small eccentricities (Goldreich \& Tremaine 1981; Ogilvie \& Lubow 2003; GS03), so a finite amplitude instability leading to eccentricity growth is a distinct possibility. Perhaps the most serious concern with the scenario by which eccentricity grows as a result of planet disk interactions involves the relative importance of eccentricity damping due to apsidal waves, although it is weaker than previously thought (GS03).

GS03 consider eccentricity evolution in the context of steady state gaps. Here we argue that conditions during gap formation are more favorable for eccentricity growth. This is significant since it is the initial stage of eccentricity growth that is the most problematic.

Next we estimate the minimal eccentricity required to saturate the corotation resonances when a gap has just formed. At this stage its width, $w \sim a / m \ll r$, is the larger of the disk's scale height and the planet's Hill radius, each of which are much smaller than the equilibrium width. Corotation saturation occurs when the density gradient is flattened over a scale $\delta=(e m \mu)^{1 / 2} r$. Our story involves the comparison of several rates, or inverse timescales, as follows:
1. The rate at which the density gradient is flattened at a first-order corotation resonance by the corotation torque,

$$
t_{\mathrm{sat}}^{-1} \approx \mu^{1 / 2} e^{1 / 2}\left(\frac{r}{w}\right)^{3 / 2} \Omega
$$

2. The rate at which viscosity reestablishes the original density gradient over a scale $\delta$,

$$
t_{\mathrm{vis}}^{-1}=\frac{\nu}{\delta^{2}} \approx \frac{\alpha}{\mu e} \frac{w}{r}\left(\frac{h}{r}\right)^{2} \Omega
$$

3. The rate at which a gap of size $w$ is opened by principal Lindblad resonances,

$$
t_{\text {gap }}^{-1} \equiv \frac{1}{w} \frac{d w}{d t} \approx \mu^{2}\left(\frac{r}{w}\right)^{5} \Omega .
$$

4. The eccentricity growth rate assuming only Lindblad resonances are active,

$$
t_{e}^{-1} \equiv \frac{1}{e} \frac{d e}{d t} \approx \mu^{2}\left(\frac{r}{w}\right)^{4} \frac{M_{d}}{M_{p}} \Omega
$$

When corotation resonances are unsaturated, eccentricity damps at a rate that is smaller by a factor of about 20 (Goldreich \& Tremaine 1980).

For the eccentricity to grow, the corotation resonances must saturate. During gap formation, this requires both $t_{\mathrm{sat}}<t_{\mathrm{vis}}$ and $t_{\text {sat }}<t_{\text {gap. }}$. Only the former is relevant for a steady state gap, and it leads to the criterion derived by Goldreich \& Tremaine (1981) and Ogilvie \& Lubow (2003). GS03 estimate a critical initial eccentricity of about $1 \%$ for eccentricity growth in equilibrium gaps, where torques at principal Lindblad resonances balance the large-scale viscous torque. However, as we now show, smaller gaps lead to less stringent constraints on the required initial eccentricity.

The requirement $t_{\mathrm{sat}}<t_{\mathrm{vis}}$ is satisfied provided

$$
e>\frac{\alpha^{2 / 3}}{\mu}\left(\frac{w}{r}\right)^{5 / 3}\left(\frac{h}{r}\right)^{4 / 3}
$$

and $t_{\text {sat }}<t_{\text {gap }}$ provided

$$
e>\mu^{3}\left(\frac{r}{w}\right)^{7}
$$

Thus, saturation is most readily achieved at a width

$$
\frac{w}{r} \sim \frac{\mu^{6 / 13}}{\alpha^{1 / 13}}\left(\frac{r}{h}\right)^{2 / 13} \sim 0.14
$$

provided the initial eccentricity satisfies

$$
e_{0}>\frac{\alpha^{7 / 13}}{\mu^{3 / 13}}\left(\frac{h}{r}\right)^{14 / 13} \sim 10^{-3}
$$

Saturation of corotation resonances is not a sufficient condition for significant eccentricity growth during gap formation. 
In addition, the growth rate of eccentricity must exceed that of the gap. This occurs for

$$
\frac{w}{r}>\frac{M_{p}}{M_{d}} \sim 0.1
$$

in other words, once the mass expelled from the gap exceeds the planet's mass. Corotation resonances are saturated at this width provided the initial eccentricity satisfies

$$
e_{0}>\frac{\alpha^{2 / 3}}{\mu}\left(\frac{h}{r}\right)^{4 / 3}\left(\frac{M_{p}}{M_{d}}\right)^{5 / 3} \sim 6 \times 10^{-4} .
$$

Significant eccentricity growth requires the initial eccentricity to satisfy both inequalities (8) and (10).

Note that eccentricity decays if corotation resonances are less than 5\% saturated. However, significant decay during gap growth can only occur once $w>20\left(M_{p} / M_{d}\right) r$. This is avoided provided the initial eccentricity satisfies the additional constraint

$$
e_{0}>10^{-12} \mu^{-4}\left(\frac{M_{d}}{M_{\odot}}\right)^{7} \sim 10^{-14}
$$

which is relevant only for lighter planets or more massive disks (e.g., a planet with mass 10 times smaller than Jupiter's in a tenth of a solar mass disk). Subsequently, we ignore the possibility of significant eccentricity decay during gap formation.

How does a planet's eccentricity grow as its gap widens after the corotation resonances are fully saturated? By dividing the gap growth rate by the eccentricity growth rate, we arrive at

$$
e=e_{0} \exp \left(\frac{w-w_{0}}{a} \frac{M_{d}}{M_{p}}\right)
$$

If the disk is 10 times more massive than the planet, by the time the gap approaches its equilibrium width, comparable to the radius of the disk, equation (12) predicts that the eccentricity would have grown by on the order of $10 e$-folds. The resulting radial excursions of the planet would cause it to pass beyond the gap edges. We expect that before this happens, additional damping that we have not modeled would come into play and limit eccentricity growth. From then on it is likely that the eccentricity would be coupled to the gap width such that $e \sim$ $w / r$. Our conclusions about eccentricity evolution as a function of gap width are depicted in Figure 1.

To conclude the discussion of corotation resonances, we mention the possibility of resonance overlap. This seems to be particularly relevant here as we are interested in eccentricity evolution when the gaps are narrow, the fractional distance between resonances, $w^{2} / r^{2}$, is small, and the fractional width of the resonances, $(\mu \mathrm{er} / \mathrm{w})^{1 / 2}$, is relatively large. Resonances overlap if

$$
e>\frac{1}{\mu}\left(\frac{w}{r}\right)^{5}
$$

The evolution of eccentricity in this regime requires further investigation. However, as shown in the figure, for our fiducial parameters most of the evolution occurs below this eccentricity, so resonance overlap probably does not play an important role even during gap formation.

Apsidal waves also promote eccentricity damping. GS03

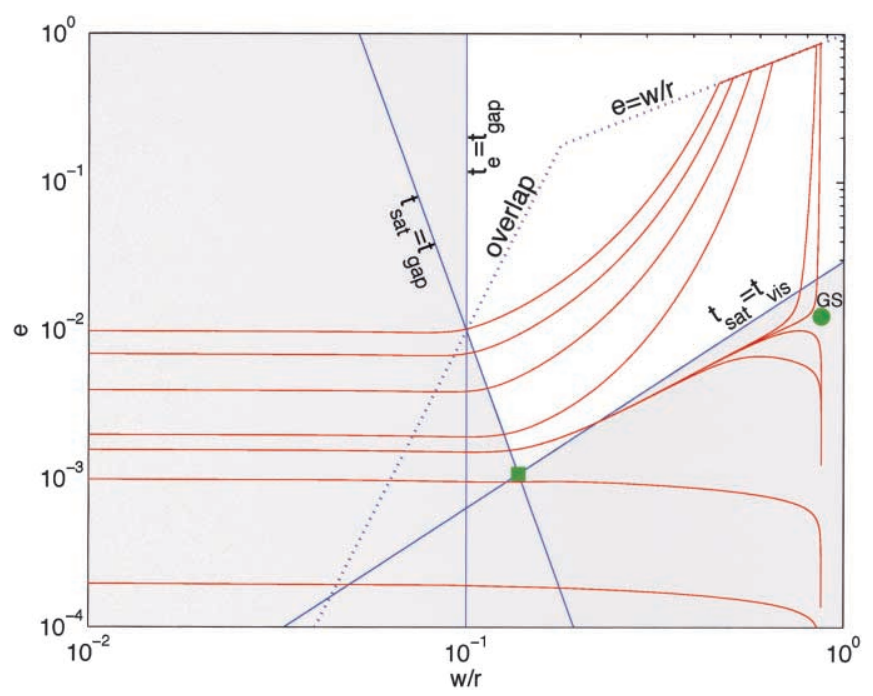

Fig. 1.-Evolution of a planet's eccentricity vs. fractional gap width for a variety of initial values of eccentricity is shown in red. Fast eccentricity growth occurs to the right of the vertical line, where the mass expelled from the gap exceeds the planet's mass, and above the two solid diagonal lines (eqs. [5] and [6]), where the corotation resonances are fully saturated. In that region of the $(e, w)$-plane, shown as unshaded, eccentricity grows exponentially until it approaches the fractional gap width shown by the dotted line marked $e=$ $w / r$. The green circle marks the criterion for eccentricity growth derived by GS03 for steady state gaps. However, since significant eccentricity growth can occur before the gap reaches its maximum width, the actual criterion is less stringent and is indicated by the green square. The criterion for resonance overlap is shown as the dotted line marked "overlap."

found their influence to be marginal for a gap at its equilibrium width. Since the relative importance of apsidal waves varies as the cube of the gap width, they are unlikely to play a major role when the gap is well below its equilibrium size. Of course, for a planet to maintain its orbital eccentricity, the apsidal waves must also be unimportant at the equilibrium gap width. But provided the eccentricity can grow when the gap is narrow, by the time the gap approaches its full width the corotation resonances will be fully saturated. Then eccentricity maintenance merely requires that eccentricity damping by apsidal waves be weaker than eccentricity excitation by first-order Lindblad resonances, a criterion that is satisfied provided

$$
\left(\frac{r}{h}\right)^{4}\left(\frac{M_{p}}{M_{*}}\right)^{2}<12
$$

(GS03). This requirement is about a factor of 20 less stringent than that derived by GS03 for the apsidal wave torque to be less important than the small difference between Lindblad and partially saturated corotation resonance torques. In particular, this looser criterion is amply satisfied by Jupiter mass planets.

Each of the rates given in equations (1)-(4) is proportional to $\Omega$ and thus decreases with distance from the star. As discussed above, eccentricity evolution depends only on the ratios of these rates. Therefore, it depends only weakly on heliocentric distance through the weak $r$-dependences of $(h / r)$ and perhaps $\alpha$. However, the rate at which the planet's mass grows imposes an additional important timescale. ${ }^{1}$ Our discussion of eccentricity growth is predicated on the assumption that planets acquire most of their mass before opening gaps. This condition

\footnotetext{
${ }^{1}$ We are indebted to R. Rafikov for raising this point.
} 
is more plausible for planets that form on large orbits than for those that form on small ones.

\section{PLANET-DISK SYMBIOSIS}

We have shown that a symbiotic relation might exist between massive planets and the disks in which they form. This relation would explain the similarity between timescales estimated to be needed for planet growth and those derived from observations of disk accretion, because disk accretion would only commence after massive planets form. In this scenario, most planets commit suicide by promoting the accretion of the disk to which they are locked. That some planets survive implies that not all of the disk material is accreted. Some other mechanism, such as evaporation, might remove the final remnants of the disks.

The interplay between eccentricity excitation and gap formation is a more subtle aspect of this symbiosis. Once a planet attains a sufficient mass, perhaps a few Earth masses, it rapidly accretes an envelope of hydrogen and helium and begins to open a gap in its disk. Provided the planet's orbit is endowed with a small initial eccentricity, the corotation resonances sat- urate while the gap width is not much larger than the disk scale height $h$. However, despite their saturation, significant eccentricity growth does not occur until the mass cleared from the gap becomes comparable to the planet's mass. Subsequently, the rate of fractional eccentricity increase exceeds that of the gap width. From then on it is plausible that the eccentricity maintains a value on the order of the fractional gap width, $e \approx w / r$. A schematic description of eccentricity and gap growth is displayed in Figure 1.

Eccentricity growth during gap opening alleviates two of the major concerns raised by Goldreich \& Sari (2003) in their discussion of possible eccentricity growth for planets in steady state gaps. It reduces both the relative importance of the apsidal torque (see eq. [14]) and the required value of the initial eccentricity (see eqs. [8] and [10]). The case for eccentricity growth due to planet disk interactions becomes more promising with this new analysis.

This research was supported in part by an NSF grant AST 00-98301 and NASA grant NAG5-12037. We thank the referee, Roman Rafikov, for comments that tightened the logic of our presentation.

\section{REFERENCES}

Artymowicz, P. 1993a, ApJ, 419, 166 1993b, ApJ, 419, 155

Goldreich, P., \& Sari, R. 2003, ApJ, 585, 1024

Goldreich, P., \& Tremaine, S. 1980, ApJ, 241, 425 . 1981, ApJ, 243, 1062

Goodman, J., \& Rafikov, R. R. 2001, ApJ, 552, 793

Hartmann, L., Calvet, N., Gullbring, E., \& D'Alessio, P. 1998, ApJ, 495, 385

Hourigan, K., \& Ward, W. R. 1984, Icarus, 60, 29

Larson, R. B. 1989, in The Formation and Evolution of Planetary Systems, ed. H. A. Weaver \& L. Danly (Cambridge: Cambridge Univ. Press), 31
Marcy, G. W., \& Butler, R. P. 1998, ARA\&A, 36, 57

Mayor, M., \& Queloz, D. 1995, Nature, 378, 355

Ogilvie, G. I., \& Lubow, S. H. 2003, ApJ, 587, 398

Rafikov, R. R. 2002, ApJ, 572, 566

Ward, W. R. 1986, Icarus, 67, 164 1997, Icarus, 126, 261

Ward, W. R., \& Hourigan, K. 1989, ApJ, 347, 490 